$3-2020$

\title{
An audit of 3-snip procedures performed at the Aga khan university hospital, Karachi, Pakistan
}

Rashid Baig

Benish Aslam

Khabir Ahmad

Follow this and additional works at: https://ecommons.aku.edu/pakistan_fhs_mc_surg_ophthalmol

Part of the Ophthalmology Commons, and the Surgery Commons 


\title{
An audit of 3-snip procedures performed at the Aga Khan University Hospital, Karachi, Pakistan
}

\author{
Rashid Baig, Benish Aslam, Khabir Ahmed
}

\begin{abstract}
Objective: To assess the functional outcome of three-snip punctoplasty procedure for punctal stenosis at a tertiary care hospital.

Methods: The retrospective study was conducted at the Aga Khan University Hospital Karachi, and comprised medical records of all patients aged $>18$ years who underwent three-snip punctoplasty between January 2013 and December 2017. Data was retrieved on age, gender, diagnosis, signs and symptoms, laterality, date of procedure, resolution of symptoms post-procedure, date of last eye followup, symptoms at last follow-up, functional outcome (epiphora at 1 month). SPSS 20 was used for data analysis.

Results: Of the 30 patients, 22(73.3\%) were females. Overall mean age at the time of punctoplasty was $57.5 \pm 15.57$ years. Of all the cases, $17(56.7 \%)$ had undergone bilateral puntoplasty. At one month, $20(66.7 \%)$ patients were completely symptom-free. Females had better results than males but the difference was not significant $(p=0.078)$.

Conclusion: Three-snip punctoplasty was found to be a minimally invasive procedure with good functional outcome that was comparable to other procedures.

Keywords: Punctal stenosis, 3-snip procedure, Epiphora, Functional outcome, Lacrimal apparatus disease, Retrospective, Aged above 18 years, Pakistan. (JPMA 70: 494; 2020).

https://doi.org/10.5455/JPMA.16265
\end{abstract}

\section{Introduction}

Punctal stenosis (PS), which is abnormal narrowing of the lacrimal punctum, was a common and treatable cause of epiphora, or watery eyes. It was often caused by inflammation. Punctoplasty, a procedure to widen the punctal opening to allow the tears to drain with ease, was indicated for excessive epiphora that was secondary to outflow obstruction from punctal stenosis. Several surgical approaches to punctoplasty have been described, the most common being the 3-snip procedure and its modern form has als been introduced.1-4 It can be further subdivided into a rectangular and triangular variation. The triangular 3-snip procedure consists of a vertical incision through the posterior wall of the punctum and vertical canaliculus, a horizontal incision along $2 \mathrm{~mm}$ of the horizontal canaliculus, and a diagonal incision connecting the start of the vertical incision with the end of the horizontal incision, resulting in a triangular excision

Aga Khan University Hospital, Karachi Pakistan.

Correspondence: Rashid Baig. e-mail: rashid.baig@aku.edu of tissue.5-9 Rectangular 3-snip procedure, also termed posterior ampullectomy, features two snips in the vertical canaliculus and a final snip at the base, with removal of the posterior wall of the ampulla.5,9

The functional outcomes of 3-snip punctoplasty for punctal stenosis has been studied in different settings in both developing and developed countries. ${ }^{10,11}$ However, there has been insufficient data from Pakistan on the outcomes of this procedure. The current study was planned to address this gap.

\section{Patients and Methods}

The retrospective study was conducted at the Aga Khan University Hospital (AKUH), Karachi, and comprised medical records of consecutive patients aged $>18$ years who underwent 3-snip punctoplasty between January 2013 and December 2017 and who had at least a onemonth follow-up. Patients with incomplete / missing medical records and those who underwent other surgical procedure for epiphora were excluded. Approval was obtained from the institutional ethics review committee. 
Table: Comparison of current data with previous studies.

\begin{tabular}{|c|c|c|c|c|c|}
\hline Author & Year & Country & Mean follow-up (in months) & Sample size & Resolution of epiphora $n(\%)$ \\
\hline Current study & 2018 & Pakistan & 1 month \& more & 47eyes $(n=30)$ & $29(66.7)$ \\
\hline Singh 1 & 2018 & India & 6 months & 34 eyes $(n=18)$ & $24(70.0)$ \\
\hline Ali 10 & 2015 & India & 6 months & 87 eyes $(n=56)$ & $65(74.7)$ \\
\hline Chak $^{5}$ & 2008 & UK & 6 days to 27.6 months & 108 eyes $(n=75)$ & $93(86.0)$ \\
\hline Caesar 11 & 2004 & UK & 1 week & $n=53^{*}$ & $49(92.0)$ \\
\hline
\end{tabular}

$\mathrm{n}=$ number, ${ }^{*}=$ number of eyes not available

Hospital information management system was used to retrieve the medical records, and a structured proforma was used to collected data on age, gender, diagnosis, signs and symptoms, laterality, date of procedure, resolution of symptoms post-procedure, date of last eye follow-up, symptoms at the last follow-up, and functional outcome in terms of epiphora at 1 month. Data was analysed using SPSS 20. Means with standard deviation (SD) were computed to describe continuous data, while frequencies and percentages were calculated to describe categorical data. $\mathrm{P}<0.05$ was considered significant.

\section{Results}

Of the 100 patients who underwent surgical correction of punctual stenosis, , 47(47\%) were excluded. Of the $53(53 \%)$ patients who underwent 3-snip punctoplasty, $30(56.6 \%)$ had a follow-up of one month or more and represented the final sample. Of them, 22(73.3\%) were females. The overall mean age at time of punctoplasty was $57.5 \pm 15.57$ years. Of all the cases, $17(56.7 \%)$ had undergone bilateral puntoplasty. At one month, 20(66.7\%) patients were completely symptom-free and $10(33.3 \%)$ didn't feel any difference. Females had better resolution compared to males but the difference was not significant $(p=0.078)$.

\section{Discussion}

The study found that epiphora at 1 month of 3-snip punctoplasty was $66.7 \%$ which makes it a good functional outcome. Multiple studies have been conducted comparing 3-snip punctoplasty with other procedures. ${ }^{12}$ One such study ${ }^{1}$ compared it with monocanalicular intubation with simple punctal dilation and showed comparable outcomes between the two procedures and the punctal dilation with monocanalicular intubation group had less re-stenosis and functional outcome was also good, but the difference was not statistically significant. In another study, 1153 patients underwent 3snip punctoplasty and $92 \%$ of them had functional success but their postoperative evaluation time was only 1 week which is a limitation. Nowadays the most commonly used technique for punctal stenosis is triangular or rectangular 3-snip punctoplasty. A 2009 study 5 found that rectangular 3-snip punctoplasty is a simple, safe, and quick procedure which maintains normal canalicular function and structure. Another study 10 also found that $82 \%$ cases showed complete resolution of symptoms post-rectangular 3-snip punctoplasty. Our findings are comparable with the studies shown in the Table.

The limitations of the current study included a small sample size and being a single-centre retrospective study, severity of punctal stenosis was also not graded and the resolution of symptoms had been self-reported. We recommend further studies to fill the gap left by the current study owing to its limitations.

\section{Conclusions}

Three-snip punctoplasty was found to be a minimally invasive procedure with a good functional outcome that was comparable to other procedures.

\section{Disclaimer: None. \\ Conflict of Interest: None. Source of Funding: None.}

\section{References}

1. Singh S, Ali MJ, Mohamed A. Comparison of outcomes of 3-snip punctoplasty versus simple punctal dilatation with monocanalicular intubation for acquired punctal stenosis. Ophthalmic Plast Reconstr Surg 2018;34:375-7.

2. Yuen KS, Chan DD, Chan WM, Lam DS. A brief history of punctoplasty: the 3-snip revisited. Eye (Lond) 2006;20:402-3.

3. Graves B. Making a new lacrimal punctum. Am J Ophthalmo 1926;9:675-7.

4. Jones LT. The cure of epiphora due to canalicular disorders, trauma and surgical failures on the lacrimal passages. Trans Am Acad Ophthalmol Otolaryngol 1962;66:506-24.

5. Chak M, Irvine F. Rectangular 3-snip punctoplasty outcomes: preservation of the lacrimal pump in punctoplasty surgery. Ophthalmic Plast Reconstr Surg 2009;25:134-5.

6. Kashkouli MB. 3-Snip punctoplasty. Eye (Lond) 2006;20:517

7. Kashkouli MB, Pakdel F. Re: "rectangular 3-snip punctoplasty outcomes: preservation of the lacrimal pump in punctoplasty surgery". Ophthalmic Plast Reconstr Surg 2010;26:221-2. 
8. Murdock J, Lee WW, Zatezalo CC, Ballin A. Three-snip punctoplasty outcome rates and follow-up treatments. Orbit 2015;34:160-3.

9. Park SJ, Noh JH, Park KB, Jang SY, Lee JW. A novel surgical technique for punctal stenosis: placement of three interrupted sutures after rectangular three-snip punctoplasty. BMC Ophthalmol 2018;18:70.

10. Ali MJ, Ayyar A, Naik MN. Outcomes of rectangular 3-snip punctoplasty in acquired punctal stenosis: is there a need to be minimally invasive? Eye (Lond) 2015;29:515-8.

11. Caesar RH, McNab AA. A brief history of punctoplasty: the 3-snip revisited. Eye (Lond) 2005;19:16-8.

12. Wong ES, Li EY, Yuen HK. Long-term outcomes of punch punctoplasty with Kelly punch and review of literature. Eye (Lond) 2017;31:560-5. 